\title{
Efeito de um programa educacional na redução de infecção da corrente sanguínea relacionada ao cateter venoso central
}

\author{
An educational program effect in reducing central line-related bloodstream infections
}

Eduardo Alexandrino S. Medeiros ${ }^{1}$

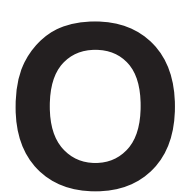

s cateteres intravasculares são dispositivos de grande importância no cenário da Unidade de Terapia Intensiva Pediátrica (UTIP). Porém, as vantagens proporcionadas pela utilização dos cateteres contrapõem-se às complicações que podem advir de seu uso, entre elas as infecções de sítio de inserção, as infecções de corrente sanguínea, a sepse, as tromboflebites e as endocardites.

Em países em desenvolvimento, incluindo o Brasil, convive-se com taxas elevadas de infecção da corrente sanguínea, principalmente em UTIs ${ }^{(1-3)}$. A infecção relacionada ao cateter venoso central é a mais frequente adquirida em UTIP e está associada ao aumento da mortalidade, do tempo de internação e custos adicionais. Parte considerável dessas infecções pode ser evitada com a aplicação de medidas de prevenção baseadas em conhecimento técnico adequado, aplicação de protocolos e apoio administrativo. Dentre essas medidas, destaca-se a higiene das mãos, que deve ser realizada com rigor, utilizando antissépticos, preferencialmente clorexidina degermante, antes da paramentação para inserção do cateter. Diversos estudos demonstram ser possível reduzir as taxas de infecção da corrente sanguínea relacionada ao cateter a valores próximos de zero com um conjunto de medidas consideradas de fácil aplicação e de baixo custo: padronização de normas para inserção do cateter, técnica estéril e cuidados pós-inserção.

O estudo de Vilela et al.(4), publicado nesta edição da Revista Paulista de Pediatria, demonstrou que a aplicação de um programa educativo interdisciplinar e o estabelecimento de normas para inserção e intervenção no processo de manutenção de cateteres reduziram significativamente a incidência de infecção da corrente sanguínea

\section{Referências bibliográficas}

1. Rosenthal VD, Maki DG, Jamulitrat S, Medeiros EA, Todi SK, Gomez DY; INICC Members. International Nosocomial Infection Control Consortium (INICC) report, data summary for 2003-2008. Am J Infect Control 2010;38:95-104.e2.

2. Arantes A, Carvalho Eda S, Medeiros EA, Farhat CK, Mantese OC. Pediatric risk of mortality and hospital infection. Infect Control Hosp Epidemiol 2004;25:783-5. relacionada a cateter. Entretanto, os autores iniciaram o estudo com taxas muito elevadas de infecção, 22,72 por mil dias de cateter e, após dois anos de intervenção, conseguiram reduzir para 5,87 infecções por mil dias de cateter. Diversos estudos brasileiros e internacionais mostram resultados semelhantes ${ }^{(5-7)}$. Pronovost et al. ${ }^{(6)}$ realizaram um estudo multicêntrico em UTIs dos Estados Unidos e demonstraram que a implementação de um conjunto de intervenções foi associada a $66 \%$ de redução na taxa de infecção da corrente sanguínea.

Para ter sucesso, um programa de prevenção de infecção necessita do envolvimento do corpo interdisciplinar da unidade, do compromisso da chefia e da aceitação das mudanças propostas na intervenção educativa. A Comissão de Controle de Infecção Hospitalar tem papel fundamental no gerenciamento das medidas de prevenção.

No estudo de Vilela et al.(4), a observação dos cuidados aos cateteres revelou problemas simples e, conforme os autores afirmam, "medidas educativas pontuais de fácil assimilação e um sistema de vigilância por processos na inserção do cateter venoso central resultaram na redução das taxas de infecção". Em relação aos agentes etiológicos, neste estudo, os Gram-positivos representaram as bactérias mais frequentemente isoladas nas infecções, semelhante ao que é observado em outros estudos realizados em UTIP. Esse fato difere do observado em UTIs de pacientes adultos, onde há maior frequência de bactérias Gram-negativas, provavelmente relacionada à quebra de técnicas no período pós-inserção do cateter ${ }^{(1,5)}$.

Os resultados do estudo de Vilela et al.(4) são facilmente reprodutíveis e devem ser utilizados como referência em programas de prevenção de infecção relacionada ao cateter em UTIP brasileiras.
3. Abramczyk ML, Carvalho WB, Carvalho ES, Medeiros EA. Nosocomial infection in a pediatric intensive care unit in a developing country. Braz $\mathrm{J}$ Infect Dis 2003;7:375-80.

4. Vilela R, Dantas SR, Trabasso P. Equipe interdisciplinar reduz a infecção sanguínea relacionada ao cateter venoso central em uma UTI pediátrica. Rev Paul Pediatria 2010;28:292-8. 
5. Santana SL, Furtado GH, Wey SB, Medeiros EA. Impact of an education program on the incidence of central line-associated bloodstream infection in 2 medical-surgical intensive care units in Brazil. Infect Control Hosp Epidemiol 2008;29:1171-3.

6. Pronovost P, Needham D, Berenholtz S, Sinopoli D, Chu H, Cosgrove S et al. An intervention to decrease catheter-related bloodstream infections in the ICU. N Engl J Med 2006;28:2725-32.

7. Guerin K, Wagner J, Rains K, Bessesen M. Reduction in central line-associated bloodstream infections by implementation of a postinsertion care bundle. Am J Infect Control 2010;38:430-3.
Instituição: Escola Paulista de Medicina da Universidade Federal de São Paulo (EPM-Unifesp), São Paulo, SP, Brasil

1 Professor Livre-Docente; Chefe da Disciplina de Infectologia do Departamento de Medicina da EPM-Unifesp e Presidente da Comissão de Controle de Infecção Hospitalar do Hospital São Paulo, São Paulo, SP, Brasi
Endereço para correspondência:

Eduardo Alexandrino S. Medeiros

Travessa Vera de Oliveira Coutinho, 116 - Paraíso

CEP: 04007-040 - São Paulo, SP

E-mail: edubala@netpoint.com.br

Conflitos de interesses: nada a declarar

Recebido em: 25/10/2010 